\title{
Longitudinal Trajectory Tracking for UGVs on Agricultural Terrains *
}

\author{
Victor R. F. Miranda* Armando Alves Neto** \\ Leonardo A. Mozelli** \\ * Programa de Pós-graduação em Eng. Elétrica, UFMG, Belo \\ Horizonte, Brasil. \\ ** Departamento de Eng. Eletrônica, UFMG, Belo Horizonte, Brasil. \\ E-mails: victormrfm@ufmg.br, \{aaneto,mozelli\}@cpdee.ufmg.br
}

\begin{abstract}
This paper presents the development of a small Unmanned Ground Vehicle (UGV) for performing payload delivery tasks in agricultural terrains. The robotic platform is equipped with low-cost navigation sensors and onboard computers, used to operate a longitudinal trajectory tracking controller. Based on the nonlinear dynamics of such vehicles, a Backstepping controller with an integral part was developed to execute these tasks. Smooth longitudinal trajectories have been calculated to be tracked by the controllers in real-time experiments. We also present a comparative performance analysis of our controller with others of the literature within different scenarios.
\end{abstract}

Keywords: Autonomous ground vehicle; Trajectory tracking; Agricultural robotics; Robust control; Nonlinear control.

\section{INTRODUCTION}

In the last decades, automation techniques have been developed to benefit several industrial, military and civilian activities. In agricultural production, for example, mobile robots have been employed to operate in crop monitoring, harvesting, maintenance of nurseries and greenhouses, among others, see Velasquez et al. (2016); Cheein and Carelli (2013). Unmanned aerial vehicles (UAVs) are commonly used in these tasks, as in Schmale III et al. (2008), but in many cases a ground viewpoint is necessary and Unmanned Ground Vehicles (UGVs) are used, see Lenain et al. (2006).

Agricultural scenarios, often require that these robots travel accurately and safely carrying extra loads across uneven and unstructured terrains. Such aspects can directly affect the performance of the UGVs during missions. Uncertainties regarding terrain irregularity and soil type can affect the speed dynamics of the robot, as well as changes in mass with the inclusion of extra loads, causing serious control problems to ensure the robot's mobility.

We have developed a small four-wheel Ackerman's UGV (shown in Fig. 1) for application in agriculture missions of payload transportation. Similar platforms can be seen in Ruiz-Larrea et al. (2016) and Oliveira et al. (2018) to perform others agricultural tasks. The developed platform is a $4 \times 4$ electric car model equipped with sensors, actuators, and an embedded computer. This is an improved version of our previous work presented in Ferreira et al. (2018).

\footnotetext{
* This work was developed with the support of CAPES (Finance Codes 001 and 88887.136349/2017-00), CNPq, FAPEMIG and PRPQ/UFMG (Institutional Research Program for Recently Hired Professors).
}

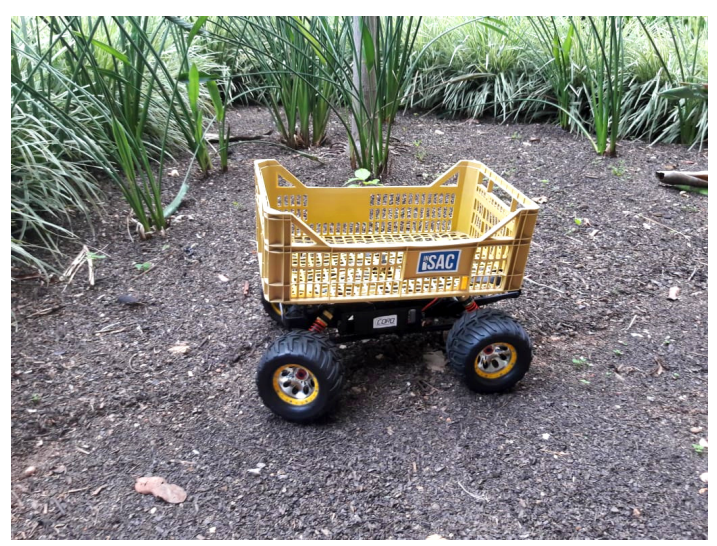

Figure 1. Our proof-of-concept car-like robotic platform employed on real-world field experiments.

Some techniques can be used to control the developed platform to follow a longitudinal trajectory, considering the nonlinearities present in such systems and enabling to reject the effects caused by the adversities of uneven terrains and/or the activity performed, as we can see in Miranda et al. (2018).

An Integral Backstepping is a control technique traditionally applied to nonlinear systems, considering the influence of some conditions in the system like the friction and inclination of the ground. The method proposed in this paper is similar to the Standard Backstepping presented in Miranda et al. (2018), using stability analysis in the Lyapunov sense to choose a control law that stabilizes the system. However, it has an integral part that seeks to guarantee null error in steady-state, as shown in Skjetne and Fossen (2004), and to minimize some disturbances provided by adverse conditions in the terrain of the trajectory. 
Our main goal is to evaluate and to compare the performances, in real-world experiments carried out on the recently developed platform, of three distinct longitudinal controllers: the Standard Backstepping and the Robust PID, presented in Miranda et al. (2018), and the Integral Backstepping, proposed in this paper. Since the simulation results in Miranda et al. (2018) indicated a superior performance regarding the PID controller, we believe that the addition of an Integral term in the Backstepping method can bridge this gap.

The real-world experiments consist of following a smooth longitudinal planned trajectory, that simulates a payload delivery mission in a farming terrain. The same mission was executed using each method to ensure that the vehicle track a virtual reference trajectory with the respective controlled speed, considering adverse conditions such as: (i) mass variation, (ii) ground slope, and (iii) soil changes.

The remainder of this paper is structured as follows: Section 2 formalizes the problem with the introduction of the dynamic model of the vehicle, followed by the design of the control law using the Integral Backstepping method, descriptions of the platform developed and the trajectories planned; Section 3 provides the real-world results using the developed platform, with respective discussions; and finally Section 4 offers the conclusion and future perspectives of the research.

\section{METHODOLOGY}

This Section describes the dynamic model of the robot, a Backstepping Integral control method applied to this model and a description of the platform designed to do real-world experiments.

\subsection{Model}

The model used in this work to represent the longitudinal dynamics of the vehicle can be determined by the set of dynamic forces acting on it and the motor transmission dynamics, as described in Miranda et al. (2018); Dias et al. (2015); Zheng et al. (2016); Gravina et al. (2017):

$$
\frac{\eta}{r} T_{\mathrm{m}}=m a+\frac{1}{2} \rho C_{D}|v| v+\operatorname{sgn}(v) \mu m|\boldsymbol{g}| \cos \theta+m|\boldsymbol{g}| \sin \theta,
$$

$$
\zeta \dot{T}_{\mathrm{m}}=\frac{T_{u}}{\beta}-T_{\mathrm{m}}
$$

where $a(t)$ is the longitudinal acceleration, $m$ is the mass of vehicle, $\rho$ is the air density, $C_{D}$ is the aerodynamic drag coefficient, $v(t)$ is the longitudinal velocity, $\operatorname{sgn}(\cdot)$ is the sign function, $|\boldsymbol{g}|$ is the modulus of gravity vector, $\theta$ is the road inclination, $\eta$ is the motor efficiency, $r$ is the wheel radius, $\zeta$ is the inertial constant, $\beta$ is the transmission ratio, $T_{\mathrm{m}}(t)$ is the torque on tires and $T_{u}(t)$ is the motor torque.

The use of the function $\operatorname{sgn}(\cdot)$ in (2) aims to evaluate the tire friction only for $v(t) \neq 0$, that is, when the vehicle starts its movement, bringing the model closer to the reality. However, $\operatorname{sgn}(\cdot)$ is discontinuous around zero and the use of an alternatively as close as possible function, but continuous throughout your domain, avoid numerical problems. Then, $\operatorname{sgn}(\cdot)$ was replaced by a similar continuous function $\left(1-e^{-v(t)}\right) /\left(1+e^{-v(t)}\right)$ in the model, without affecting dynamics.

Rewriting (2) in the states space model, we have the nonlinear system:

$$
\left\{\begin{aligned}
\dot{\boldsymbol{x}}_{1} & =\boldsymbol{x}_{2} \\
\dot{\boldsymbol{x}}_{2} & =\frac{1}{m}\left(\frac{\eta}{r} \boldsymbol{x}_{3}-\frac{1}{2} \rho C_{D}\left|\boldsymbol{x}_{2}\right| \boldsymbol{x}_{2}-m|\boldsymbol{g}| \sin \theta\right. \\
& \left.-\frac{1-e^{-x_{2}}}{1+e^{-x_{2}}} \mu m|\boldsymbol{g}| \cos \theta\right) \\
\dot{\boldsymbol{x}}_{3} & =\frac{1}{\zeta}\left(u-\boldsymbol{x}_{3}\right),
\end{aligned}\right.
$$

where $\boldsymbol{x}_{1}(t)$ is the longitudinal position, $\boldsymbol{x}_{2}(t)$ is the longitudinal velocity, $\boldsymbol{x}_{3}(t)$ is the torque and $u(t)=$ $T_{u}(t)$ is the torque command. It was considered a direct transmission from the motor to the wheels, $\beta=1$.

\subsection{Backstepping Integral}

The Backstepping is a classic control technique used in stability control of systems with nonlinear dynamics. In this technique, the Lyapunov stability theory is used to choose a control law such that the studded system becomes stable (Khalil and Grizzle, 2002; Krstic et al., 1995).

This section presents a Backstepping controller with an integral action to determine a control law to the nonlinear system (3) such that the error between a virtual reference trajectory and the position of the robot $\left[x_{r}(t)-x_{1}(t)\right]$ become zero in steady-state.

The integral Backstepping controller design starts following the standard method as in Khalil and Grizzle (2002). Thus, it is necessary to make a change of variables in the system (3) to format it as a superior triangular system. As in Miranda et al. (2018), let

$$
u=\zeta u_{n}+x_{3},
$$

then

$$
\dot{\boldsymbol{x}}_{3}=u_{n}
$$

Based in the procedure seen in Skjetne and Fossen (2004),Mian et al. (2008) and Rashad et al. (2015), this method is formulated to solve the tracking problem $\lim _{t \rightarrow \infty}\left[x_{r}(t)-x_{1}(t)\right]=0$ in three steps as follows:

\section{- First Step}

In the first step is chosen the Backstepping states transformation as: $z_{1}(t)=x_{r}(t)-x_{1}(t)$ and $\xi(t)=\int_{0}^{t} z_{1}(\tau) d \tau$, where $z_{1}(t)$ is the error of position and $\xi(t)$ is the integral term of error. The dynamics after these transformations is:

$$
\begin{aligned}
\dot{\xi}(t) & =z_{1}(t), \\
\dot{z}_{1}(t) & =\dot{x}_{r}(t)-x_{2}(t) .
\end{aligned}
$$

The following Lyapunov function is proposed to guarantee the stability of the system in (6) ${ }^{1}$ :

$$
V_{1}\left(\xi, z_{1}\right)=\frac{1}{2} K \xi^{2}+\frac{1}{2} z_{1}^{2},
$$

where $K>0$ and, consequently, $V_{1}\left(\xi, z_{1}\right)>0, \forall\left[\xi z_{1}\right] \neq 0$.

\footnotetext{
1 From this point, the time dependence (t) will be suppressed.
} 
According to Khalil and Grizzle (2002) and Krstic et al. (1995), to guarantee the stability of the system, the derivative of Lyapunov function needs to be negative semidefinite. In this way, for

$$
\begin{aligned}
& \dot{V}_{1}\left(\xi, z_{1}\right)=K \xi \dot{\xi}+z_{1} \dot{z}_{1}, \\
& \dot{V}_{1}\left(\xi, z_{1}\right)=z_{1}\left(K \xi+\dot{x}_{r}-x_{2}\right) .
\end{aligned}
$$

Choosing $\phi_{1}=x_{2}$ and $\phi_{1}=K \xi+\dot{x}_{r}+C_{1} z_{1}$, with $C_{1}>0$. Replacing in (8):

$$
\begin{aligned}
& \dot{V}_{1}\left(\xi, z_{1}\right)=z_{1}\left(K \xi+\dot{x}_{r}-\phi_{1}\right), \\
& \dot{V}_{1}\left(\xi, z_{1}\right)=-C_{1} z_{1}^{2}<0,
\end{aligned}
$$

which is negative definite and guarantees the asymptotic stability of the system (6).

\section{- Second Step}

Adding and subtracting $\phi_{1}$ in the second equation of (6) we have:

$$
\begin{aligned}
\dot{\xi} & =z_{1} \\
\dot{z}_{1} & =\dot{x}_{r}-\phi_{1}+\left(\phi_{1}-x_{2}\right)=-K \xi-C_{1} z_{1}+\left(\phi_{1}-x_{2}\right) .
\end{aligned}
$$

Making a change of variables $z_{2}=x_{2}-\phi_{1}$, a new system can be rewritten as:

$$
\begin{aligned}
\dot{\xi} & =z_{1} \\
\dot{z}_{1} & =\dot{x}_{r}-\phi_{1}+\left(\phi_{1}-x_{2}\right)=-K \xi-C_{1} z_{1}-z_{2}, \\
\dot{z}_{2} & =\left(\frac{\eta}{m r} x_{3}-\frac{1}{2} \rho C_{D}\left|x_{2}\right| x_{2}-|\boldsymbol{g}| \sin \theta\right. \\
& \left.-\frac{1-e^{-x_{2}}}{1+e^{-x_{2}}} \mu|\boldsymbol{g}| \cos \theta\right)-\dot{\phi}_{1}
\end{aligned}
$$

where

$$
\dot{\phi}_{1}=K z_{1}+\ddot{x}_{r}-C_{1}\left(z_{2}+K \xi+C_{1} z_{1}\right) .
$$

Consider a new Lyapunov function as

$$
V_{2}\left(\xi, z_{1}, z_{2}\right)=V_{1}\left(\xi, z_{1}\right)+\frac{1}{2} z_{2}^{2}>0, \forall\left[\begin{array}{lll}
\xi & z_{1} & z_{2}
\end{array}\right] \neq 0,
$$

which has the following derivative:

$$
\dot{V}_{2}\left(\xi, z_{1}, z_{2}\right)=K \xi \dot{\xi}+z_{1} \dot{z}_{1}+z_{2} \dot{z}_{2},
$$

Then, to guarantee the asymptotic stability, it is necessary a virtual control law $\phi_{2}$, in this case will be $x_{3}$, which makes $\dot{V}_{2}\left(\xi, z_{1}, z_{2}\right)<0$. Therefore,

$$
\begin{aligned}
\phi_{2} & =\frac{m r}{\eta}\left(\frac{1}{2} \rho C_{D}\left|x_{2}\right| x_{2}+|\boldsymbol{g}| \sin \theta+\frac{1-e^{-x_{2}}}{1+e^{-x_{2}}} \mu|\boldsymbol{g}| \cos \theta\right. \\
& \left.+\dot{\phi}_{1}+z_{1}-C_{2} z_{2}\right)
\end{aligned}
$$

and replacing in (14) we have $\dot{V}_{2}<0 \forall\left[\begin{array}{ll}z_{1} & z_{2}\end{array}\right]$ since that $C_{1}>0$ and $C_{2}>0$.

$$
\dot{V}_{2}\left(\xi, z_{1}, z_{2}\right)=-C_{1} z_{1}^{2}-C_{2} z_{2}^{2}<0 .
$$

\section{- Third Step}

Following the same approach used in the last step, a change of variables is made as $z_{3}=x_{3}-\phi_{2}$ and a new system can be rewritten including $z_{3}$ dynamic as:

$$
\begin{aligned}
\dot{\xi} & =z_{1}, \\
\dot{z}_{1} & =-z_{2}-K \xi-C_{1} z_{1}, \\
\dot{z}_{2} & =\frac{\eta}{m r} z_{3}+z_{1}-C_{2} z_{2}, \\
\dot{z}_{3} & =u_{n}-\dot{\phi}_{2},
\end{aligned}
$$

where

$$
\begin{aligned}
\dot{\phi}_{2} & =\frac{m r}{\eta}\left[\rho C_{D}\left|x_{2}\right| x_{2}+|\boldsymbol{g}| \sin \theta+\mu|\boldsymbol{g}| \cos \theta \frac{2 e^{-x_{2}}}{\left(1+e^{-x_{2}}\right)^{2}} \dot{x}_{2}\right. \\
& \left.+\dot{z}_{1}(K+1)+\dddot{x}_{r}-C_{1}\left(\dot{z}_{2}+K \xi+C_{1} z_{1}\right)-C_{2} \dot{z}_{2}\right] .
\end{aligned}
$$

A new Lyapunov function it is necessary to evaluate the system stability. Thus,

$$
V_{3}\left(\xi, z_{1}, z_{2}, z_{3}\right)=V_{2}\left(\xi, z_{1}, z_{2}\right)+\frac{1}{2} z_{3}^{2},
$$

where $V_{3}\left(\xi, z_{1}, z_{2}, z_{3}\right)>0 \forall\left[\begin{array}{llll}\xi & z_{1} & z_{2} & z_{3}\end{array}\right] \neq 0$.

To be asymptotically stable, the derivative of the Lyapunov function needs to be negative definite. In this way, it is necessary a control law that guarantees $\dot{V}_{3}<0$. Being $\dot{V}_{3}\left(\xi, z_{1}, z_{2}, z_{3}\right)=-C_{1} z_{1}^{2}-C_{2} z_{2}^{2}+\frac{\eta}{m r} z_{2} z_{3}+z_{3}\left(u_{n}-\dot{\phi}_{2}\right)$,

let choose

$$
u_{n}=\dot{\phi}_{2}-\frac{\eta}{m r} z_{2}-C_{3} z_{3}
$$

such that, by replacing (21) in (20),

$$
\dot{V}_{3}\left(\xi, z_{1}, z_{2}, z_{3}\right)=-C_{1} z_{1}^{2}-C_{2} z_{2}^{2}-C_{3} z_{3}^{2} .
$$

Therefore, by inspection $\dot{V}_{3}<0, \forall\left[z_{1}, z_{2}, z_{3}\right] \neq 0$, since that $\left[C_{1}, C_{2}, C_{3}\right]>0$. Then, according the Lemma present in Khalil and Grizzle (2002); Krstic et al. (1995) and as show in Miranda et al. (2018), replacing (21) into (4), the system (3) will be globally asymptotically stable for a bounded reference input $x_{r}$ and guarantees that $\lim _{t \rightarrow \infty}\left[x_{r}(t)-x_{1}(t)\right]=0$, since $\left[K, C_{1}, C_{2}, C_{3}\right]>0$.

\subsection{Platform Description}

To execute farm tasks, a small Ackermann-based robot was built over the Mad Force Kruiser 2.0 1/8 Monster Truck platform (Fig. 1). This robot is a new version of that was used in Ferreira et al. (2018), where all system was computed in a Smartphone with Android OS.

In this paper, the platform was built with a Raspberry Pi 3 computer with Ubuntu Mate 18.04 to do data processing and control actions executing a framework designed in Robot Operating System (ROS) ${ }^{2}$, presented in Fig. 2, with algorithms implemented in the Python programming language.

The feedback information is provided by a digital incremental rotary encoder LPD3806-360BM, coupled in an intermediate reduction between the motor's shaft and the wheel, firstly handled by an Arduino Nano microcontroller, which calculates the speed and position, sending them via serial communication to the Raspberry.

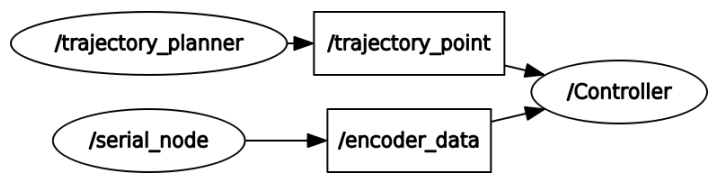

Figure 2. Control architecture at the ROS.

The torque command is ensured by an Electronic Speed Controller $(E S C)$, operated via $P W M$ inputs come from

2 www.ros.org. 


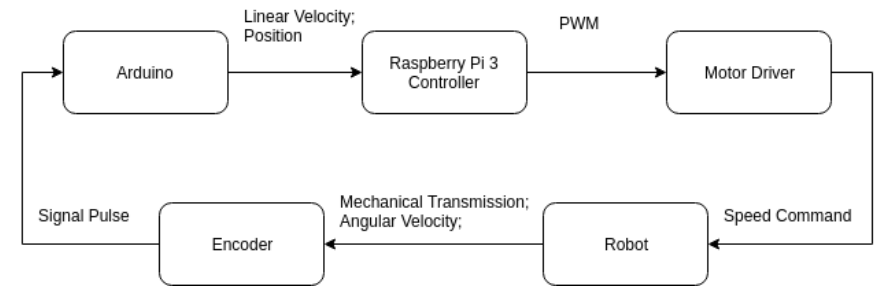

Figure 3. Flowchart of the control operation in the Platform.

the Raspberry and pass through an Adafruit PCA9685 motors driver before it reaches the ESC. The control input used in the model is the torque applied to the motor (N.m), thus it was made a previous calibration to relate the two quantities using a digital torque sensor and the least square method to estimate. This relationship is different in the forward and reverse directions of the engine speed and is given by

$$
\begin{aligned}
P W M_{\text {forward }} & =379.02+4.21\left(T_{\text {forward }}\right), \\
P W M_{\text {reverse }} & =353.69-10.86\left(T_{\text {reverse }}\right),
\end{aligned}
$$

where $\mathrm{T}$ is the motor torque and PWM is the value sent to the ESC.

With this platform it is possible to perform payload delivery tasks, for example, from a control operation that follows the flowchart in Fig. 3.

\subsection{Trajectory Planning}

The trajectory to be followed for the robot is based only in longitudinal movements and the basic problem of the generation is to move the robot from an initial position to a final position. In general, we can represent a trajectory as a polynomial-time function which the order depends on the number of constraints inserted in the planning. For the experiments that were performed, third-order polynomial functions were used.

These trajectories are formed by an initial position $x_{0}$, a final position $x_{f}$ and the time $t_{f}$ spent to travel from $x_{0}$ to $x_{f}$, obeying four constraints: $x(0)=x_{0}, x\left(t_{f}\right)=x_{f}$, $\dot{x}(0)=0$ and $\dot{x}\left(t_{f}\right)=0$. Such constraints are satisfied by the cubic polynomial function:

$$
\left\{\begin{array}{l}
x(t)=a_{0}+a_{1} t+a_{2} t^{2}+a_{3} t^{3} \\
\dot{x}(t)=a_{1}+2 a_{2} t+3 a_{3} t^{2}
\end{array}\right.
$$

where $a_{0}=x_{0}, a_{1}=0, a_{2}=\frac{3}{t_{f}^{2}}\left(x_{f}-x_{0}\right)$ and $a_{3}=$ $-\frac{2}{t_{f}^{3}}\left(x_{f}-x_{0}\right)$. The main advantage of using smooth trajectories at this application is the avoiding of abrupt jerks in the vehicle's engine.

\section{RESULTS}

In this section, we present real-world results of the Integral Backstepping controller in Section 2.2, applied to the platform developed present in Section 2.3 and comparing them with the results of the Standard Backstepping and Robust PID show in Miranda et al. (2018) applied to the same platform.

The parameters describes in Table 1, which refer to the developed platform, was used to calculate the Backstepping control law (4) for perform the experiments.
Table 1. Model parameters used in the Backstepping method.

\begin{tabular}{ccc}
\hline Parameter & Value & Unity \\
\hline Mass $(m)$ & 5.50 & $\mathrm{Kg}$ \\
Gravity $(|\boldsymbol{g}|)$ & 9.81 & $\mathrm{~m} / \mathrm{s}^{2}$ \\
Air density $(\rho)$ & 1.18 & $\mathrm{Kg} / \mathrm{m}^{3}$ \\
Drag coeff. $\left(C_{D}\right)$ & 0.10 & {[]} \\
Wheel radius $(r)$ & 0.08 & $\mathrm{~m}$ \\
Motor efficiency $(\eta)$ & 95.00 & $\%$ \\
Road angle $(\theta)$ & 0.00 & $\mathrm{rad}$ \\
Torque inertia $(\zeta)$ & 0.10 & {[]} \\
\hline
\end{tabular}

The purpose of the experiments is to reflect a typical farming mission of payload delivery. Thus, the robot must track a smooth longitudinal trajectory that promotes a displacement in both directions (forward and backward), in different terrain types, with slopes and with extra loads, represented by two dumbbells of $1 \mathrm{~kg}$, added to the vehicle during some stops. In these experiments, we seek to evaluate the effect of these adverse conditions on the system and the performance of each controller. The steering of the front wheels was fixed using the procedure from Ferreira et al. (2018), such that the robot follows the planned longitudinal trajectory semi-autonomously.

Firstly, two missions have been tested, which are described in Table 2. All controllers are tested in the same mission and the results are shown in Figures 4 and 5, which represents the trajectory traveled and the speed employed in the mission 1 and 2 , respectively.

Table 2. Scenarios of missions: mass variation, slope profile and terrain type.

\begin{tabular}{c|cccc}
\hline \multirow{2}{*}{ Scenario } & \multicolumn{2}{|c}{$\begin{array}{c}m[\mathrm{~kg}] \\
(\text { mission })\end{array}$} & slope $\approx 20^{\circ}$ & ground-type \\
\cline { 2 - 3 } & 1 & 2 & & \\
\hline S1 & 5.5 & 7.5 & no slope & regular stone \\
S2 & 5.5 & 7.5 & climbing & tall grass \\
S3 & 6.5 & 6.5 & climbing & tall grass \\
S4 & 7.5 & 5.5 & descending & tall grass \\
S5 & 7.5 & 5.5 & no slope & regular stone \\
\hline
\end{tabular}

Another experiment was performed in a bumpy dirt road, submitting the robot to a path with holes and small obstacles as: sticks, stones, and leaves. The same trajectory was passed as a reference to the controllers and the mission scenarios are describe in Table 3. The exactly ground inclination present in these experiments is unknown, but it is known that is smaller (almost zero) than the missions in Table 2. The results are shown in Figures 6 and 7.

Table 3. Scenario description of the experiments on the bumpy road.

\begin{tabular}{c|cc}
\hline \multirow{2}{*}{ Scenario } & \multicolumn{2}{|c}{$m[\mathrm{~kg}]$} \\
\cline { 2 - 3 } & $($ mission 1$)$ & $($ mission 2$)$ \\
\hline S1 & 5.5 & 7.5 \\
S2 & 6.5 & 6.5 \\
S3 & 7.5 & 5.5 \\
\hline
\end{tabular}

In adverse situations, presented in all missions of the experiments, even with null error in steady-state, it is possible to notice that the integral Backstepping controller has more difficulties to reject the disturbances (ground slopes) and the uncertainties of the parameters (change of mass and soil type) than the Robust PID. It is clear that 

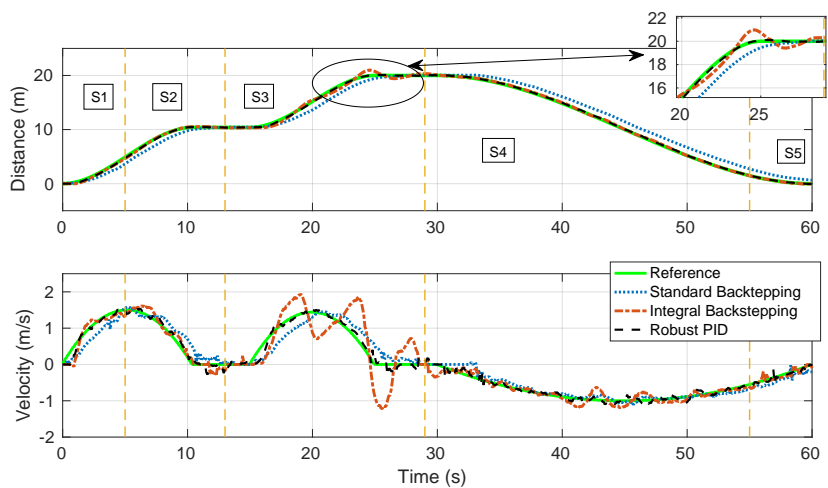

Figure 4. Results of mission 1 (Table 2): position and speed of Reference and all tested controllers.
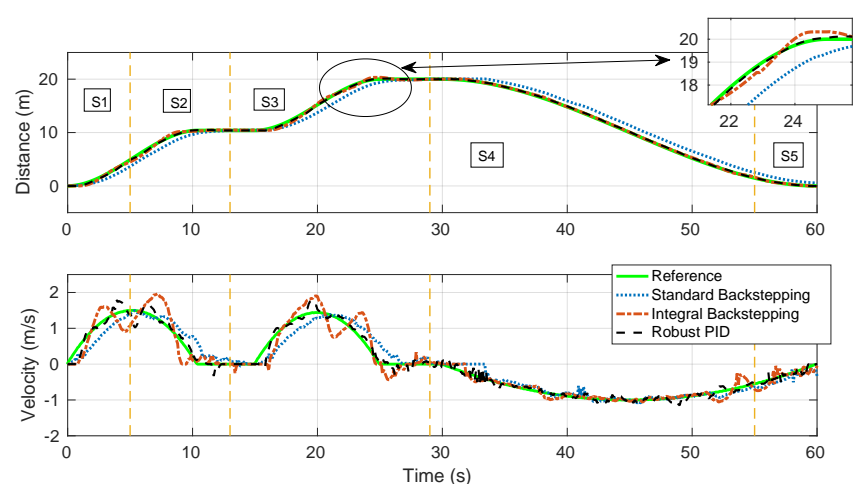

Figure 5. Results of mission 2 (Table 2): position and speed of Reference and all tested controllers.
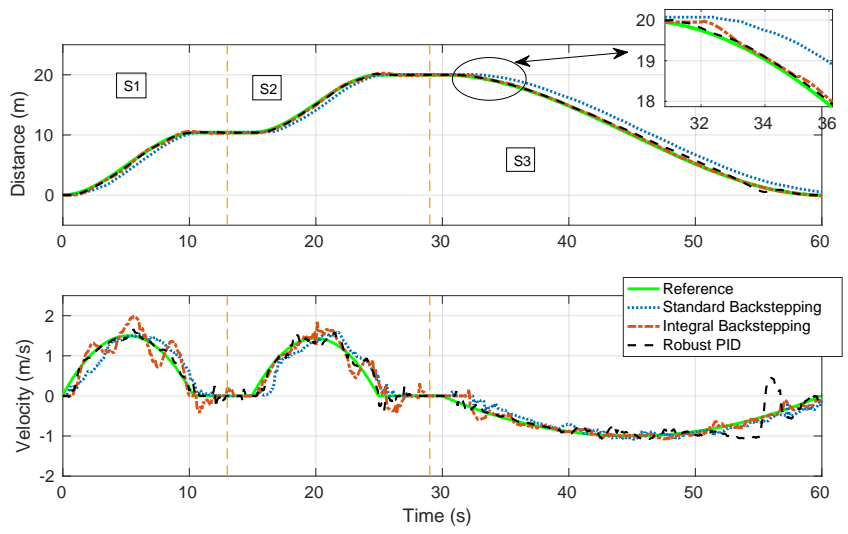

Figure 6. Results of mission 1 (Table 3 ): position and speed of Reference and all tested controllers.

the PID was designed to be robust to these adversities, but the integral Backstepping takes on the nonlinearities of the system different from the PID, which uses the linearized model. Therefore, even using a more complete model, the Integral controller demonstrates a worse performance than the Robust PID.

The difference of the controller's performance was evaluated using the IAE (Integral Absolute Error), ITAE (Integral Time Absolute Error), ISE (Integral Square Error) and ITSE (Integral Time Square Error) indexes. The Table 4 shows a mean of these indexes for each controller in all
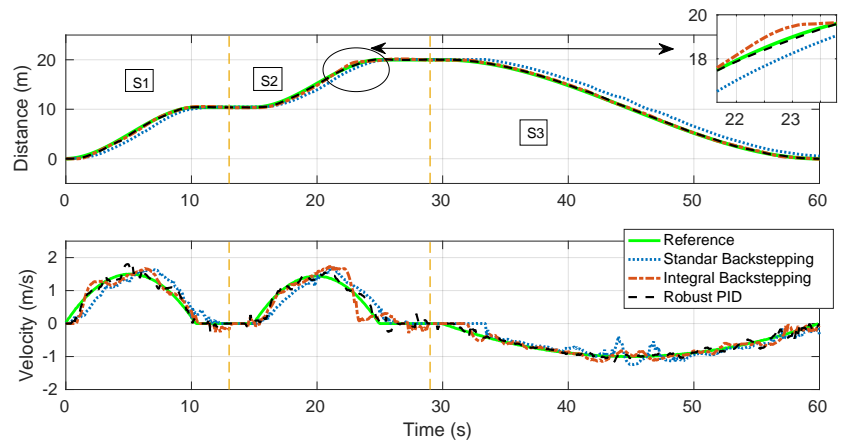

Figure 7. Results of mission 2 (Table 3): position and speed of Reference and all tested controllers.

missions of the experiments, calculated by the equations:

$$
\begin{aligned}
& I A E=\int_{0}^{T}|e(t)| d t ; \quad I T A E=\int_{0}^{T} t|e(t)| d t ; \\
& I S E=\int_{0}^{T} e(t)^{2} d t ; \quad I T S E=\int_{0}^{T} t e(t)^{2} d t .
\end{aligned}
$$

These indexes evaluate the performance of the controllers in converging to a proposed reference and the time spent in this convergence. Observing the indexes, it is possible to notice that the Robust PID has the lowest values for them, what translate the better results of performance. Also, it is possible to notice the difficulty of the standard Backstepping in converges to the reference, as in the graphic analysis.

Table 4. Average performance indices from the experiments for each controller.

\begin{tabular}{c|cccc}
\hline Controller & IAE & ITAE & ISE & ITSE \\
\hline Standard Backstepping & 50.47 & $1,759.20$ & 116.84 & 120.73 \\
Integral Backstepping & 8.13 & 198.42 & 4.19 & 4.28 \\
Robust PID & 5.79 & 174.83 & 2.12 & 2.14 \\
\hline
\end{tabular}

\section{CONCLUSIONS}

This work addresses the problem of controlling a ground robot in uneven terrains, typically of an agricultural environment. Three controllers were compared in real-world missions of payload delivery in longitudinal trajectories, using a small designed platform. The experimental results showed that the integral Backstepping controller and the robust PID can reduce the tracking error and, even using a simplified linear model, the PID rejected better the disturbances and the uncertainties with a simpler tuning method. Therefore, it can be concluded that the uncertainty in the parameters and the presence of disturbances have a strong influence on the system. Besides, the Backstepping integral controller can reduce the influence of these adverse operating conditions while minimizing the tracking error. The inclusion of an integral action promotes a significant improvement in the performance indexes evaluated, of some orders of magnitude, when compared to the standard Backstepping version.

The developed platform obtained a satisfactory performance in executing the control systems during the experiments. In addition, the platform structure can be general- 
ized to any type of car-like four-wheeled vehicle, and the control system can be used to trajectory tracking.

As future work, we intend to study a method to robust the integral Backstepping to the uncertainties and disturbances. We also intend to study the lateral-directional dynamics of our robot to provide a two-dimensional trajectory tracking to the system in uneven terrain conditions. With this, the platform must be improved with new sensors to estimate lateral displacements and orientation of the robot. We may also incorporate a state estimation filter to improve the measurement since encoders often introduce dead-reckoning errors in the navigation.

\section{REFERENCES}

Cheein, F.A.A. and Carelli, R. (2013). Agricultural robotics: Unmanned robotic service units in agricultural tasks. IEEE Industrial Electronics Magazine, 7(3), 4858.

Dias, J.E.A., Pereira, G.A.S., and Palhares, R.M. (2015). Longitudinal model identification and velocity control of an autonomous car. IEEE Trans. on Intelligent Transportation Systems, 16(2), 776-786.

Ferreira, E., Miranda, V., Silva Junior, M., Mozelli, L., and AlvesS Neto, A. (2018). Aplicação de plataforma android no controle de robôs móveis para inspeção de lavouras. Congresso Brasileiro de Automática - $C B A$ 2018.

Gravina, A.P.G., Mozelli, L.A., and Souza, F.O. (2017). Estudo do efeito do atraso de comunicaçao em comboio de veıculos autônomos. In Simpósio Brasileiro de Automação Inteligente, 25-30. Porto Alegre, RS.

Khalil, H.K. and Grizzle, J. (2002). Nonlinear systems, vol. 3, volume 3 .

Krstic, M., Kanellakopoulos, I., and Kokotovic, P.V. (1995). Nonlinear and adaptive control design. Wiley.

Lenain, R., Thuilot, B., Cariou, C., and Martinet, P. (2006). High accuracy path tracking for vehicles in presence of sliding: Application to farm vehicle automatic guidance for agricultural tasks. Autonomous robots, 21(1), 79-97.
Mian, A.A., Ahmad, M.I., and Wang, D. (2008). Backstepping based pid control strategy for an underactuated aerial robot. IFAC Proceedings Volumes, 41(2), 1563615641.

Miranda, V., Alves Neto, A., and Mozelli, L.A. (2018). Estudo sobre estratégias de controle longitudinal para robôs terrestres em terrenos irregulares com inclinação. Congresso Brasileiro de Automática - CBA 2018.

Oliveira, A.I., Prado, M.G., and Leite, A.C. (2018). Adaptação de um automodelo para aplicações de robótica móvel na agricultura. Congresso Brasileiro de Automática - CBA 2018.

Rashad, R., Aboudonia, A., and El-Badawy, A. (2015). Backstepping trajectory tracking control of a quadrotor with disturbance rejection. In Int. Conf. on Information, Communication and Automation Technologies (ICAT), 1-7. IEEE.

Ruiz-Larrea, A., Roldán, J.J., Garzón, M., del Cerro, J., and Barrientos, A. (2016). A ugv approach to measure the ground properties of greenhouses. In Robot 2015: Second Iberian Robotics Conf., 3-13. Springer.

Schmale III, D.G., Dingus, B.R., and Reinholtz, C. (2008). Development and application of an autonomous unmanned aerial vehicle for precise aerobiological sampling above agricultural fields. Journal of Field Robotics, 25(3), 133-147.

Skjetne, R. and Fossen, T.I. (2004). On integral control in backstepping: Analysis of different techniques. In American Control Conf., volume 2, 1899-1904. IEEE.

Velasquez, A., Higuti, V., Borrero Guerrero, H., Milori, D., Magalhães, D., and Becker, M. (2016). Helvis - a smallscale agricultural mobile robot prototype for precision agriculture. In Proc. of the 13th Int. Conf. on Precision Agriculture, 1-17. St. Louis, USA.

Zheng, Y., Li, S.E., Wang, J., Cao, D., and Li, K. (2016). Stability and scalability of homogeneous vehicular platoon: Study on the influence of information flow topologies. IEEE Trans. on Intelligent Transportation Systems, 17(1), 14-26. 